\title{
Rotordynamic Forces on a Four Bladed Inducer
}

\author{
Angelo Pasini ${ }^{1}$, Lucio Torre $^{2}$, Angelo Cervone ${ }^{3}$ and Luca d'Agostino ${ }^{4}$ \\ ALTA S.p.A. - Via Gherardesca, 5 - 56121 Ospedaletto, Pisa, Italy
}

The present paper illustrates the results of an experimental campaign conducted in the CPRTF (Cavitating Pump Rotordynamic Test Facility) at ALTA S.p.A. aimed at characterizing the rotordynamic forces acting on a whirling four-bladed, tapered-hub, variable-pitch inducer, designated as DAPAMITO4. The forces acting on the impeller have been measured by means of a rotating dynamometer mounted just behind the inducer. The roles of the imposed whirl motion of the rotor, flow coefficient, cavitation number and liquid temperature have been investigated. The destabilizing role of cavitation has been confirmed. The experimental results are consistent with previous findings obtained by the authors, as well as with former data published by Caltech researchers. The observed dependence of the tangential and normal components of the rotordynamic force on the whirl-to-rotational speed ratio does not follow the quadratic functional behavior often assumed in the open literature. Rotordynamic forces of large amplitude and destabilizing nature especially occur in the presence of cavitation, potentially compromising the stability of the pump operation.

\section{Nomenclature}

$\begin{array}{ll}A & \text { rotordynamic matrix } \\ A & \text { cross-section area } \\ A_{i j} & \text { element of the rotordynamic matrix } \\ c_{a} & \text { full-blade axial length } \\ c_{\%} & \text { tip clearance/mean blade height ratio } c_{\%}=\delta / h_{m} \\ D & \text { diffusion factor } \\ F & \text { generalized dimensional force } \\ F^{*} & \text { generalized nondimensional force } \\ I, J & \text { integer numbers } \\ L & \text { axial length } \\ N & \text { number of blades } \\ p & \text { static pressure } \\ Q & \text { volumetric flow rate } \\ R e & \text { Reynolds number } \operatorname{Re}=2 \Omega r_{T}^{2} / v \\ r_{H} & \text { inducer hub radius } \\ r_{T} & \text { inducer tip radius }\end{array}$

${ }^{1}$ Ph.D. Student, Aerospace Engineering Department, Pisa University - Project Engineer, ALTA S.p.A., Pisa, Italy, AIAA Member; a.pasini@alta-space.com

${ }^{2}$ Project Manager, ALTA S.p.A., Pisa, Italy, AIAA Member; 1.torre@alta-space.com

${ }^{3}$ Project Manager, ALTA S.p.A., Pisa, Italy, AIAA Member; a.cervone@ alta-space.com

${ }^{4}$ Professor, Aerospace Engineering Department, Pisa University - Vice President, ALTA S.p.A., Pisa, Italy, AIAA Member; luca.dagostino@ing.unipi.it 


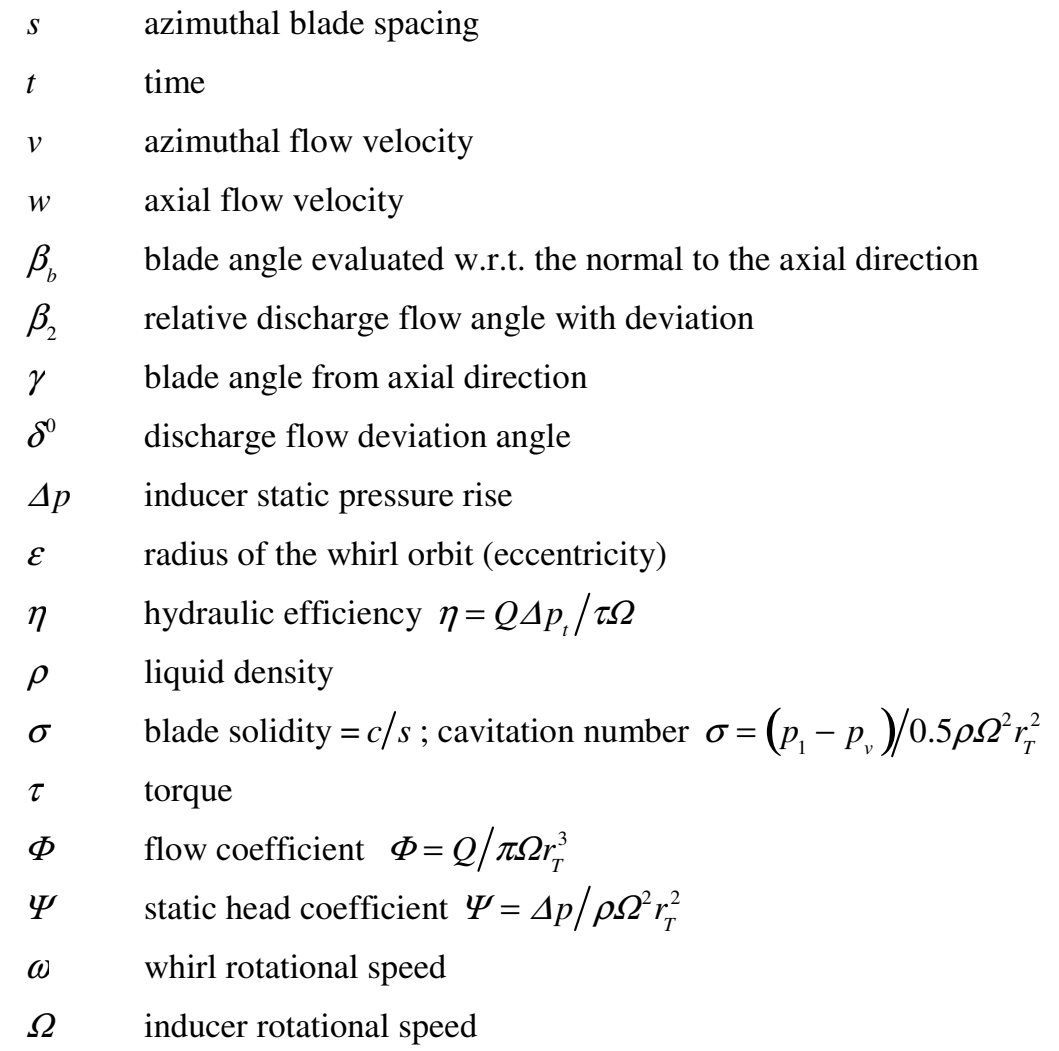

\section{$\underline{\text { Subscripts }}$}

$D \quad$ design conditions

$N \quad$ normal to the whirl orbit

$T \quad$ tip radius/ tangent to the whirl orbit

$H \quad$ hub radius

$x, y$ rotating reference frame

$X, Y \quad$ absolute reference frame

le blade leading edge

te blade trailing edge

$v \quad$ vapor pressure

$\delta^{\circ} \quad$ flow deviation angle

$0 \quad$ initial condition/ steady forces

1 inlet section

2 outlet section

\section{Introduction}

$\mathbf{R}$ otordynamic forces, together with flow instabilities generated or not by cavitation, are one of the universally R recognized and most dangerous sources of vibrations in turbomachines. These forces can affect all of the components of the machine, including the bearings, the seals and, obviously, the impeller itself (Ehrich \& Childs ${ }^{1}$ ).

The rotordynamic forces acting on centrifugal pumps have been measured and studied in the past (see for example Hergt \& Krieger $^{2}$; Ohashi \& Shoji ${ }^{3}$ ), even if only limited information is available on their dependence on cavitation $\left(\right.$ Jery $^{4} ;$ Franz $^{5}$ ). Recently Yoshida et al. $^{6}$ have investigated the effects of seal geometry on the 
rotordynamic forces acting on a simple two-dimensional centrifugal impeller with logarithmic spiral blades, while Suzuki et al. ${ }^{7}$ have measured the rotordynamic forces on an artificial heart pump impeller. Experimental data on the influence of cavitation on radial and rotordynamic forces on turbopump impellers mainly come from the work carried out at the California Institute of Technology, Pasadena, California, USA, in the context of the doctoral theses of Jery ${ }^{4}$, Franz ${ }^{5}$ and Bhattacharyya ${ }^{8}$. In particular, at present the rotordynamic forces acting on axial inducers, especially in the unshrouded configurations currently used for space rockets applications, are not yet perfectly understood.

The rotordynamic configuration of the Cavitating Pump Test Facility (CPTF) at ALTA S.p.A. (Rapposelli et al. ${ }^{10}$ ) is specifically intended for the analysis of steady and unsteady fluid forces and moments acting on the impeller as a consequence of its whirl motion under cavitating or fully-wetted flow conditions, with special emphasis on the onset and development of lateral rotordynamic instabilities. The present paper illustrates the results of an experimental campaign carried out at ALTA S.p.A., Pisa, Italy, on a four bladed inducer, aimed at investigating the influence of cavitation on rotordynamic forces by means of forced vibration experiments where the impeller is subject to a whirl motion of given constant eccentricity and angular velocity.

\section{Experimental Apparatus}

\section{A. Test Facility and Instrumentation}

The experimental activity reported in the present paper has been carried out using ALTA's Cavitating Pump Rotordynamic Test Facility (CPRTF, Figure 1), specifically designed for characterizing the performance of cavitating/non-cavitating turbopumps in a wide variety of alternative configurations (axial, radial or mixed flow, with or without an inducer; Rapposelli et al. ${ }^{10}$ ). The facility operates in water at temperatures up to $90{ }^{\circ} \mathrm{C}$ and is intended as a flexible apparatus readily adaptable to conduct experimental investigations on virtually any kind of fluid dynamic phenomena relevant to high performance turbopumps. The inlet section, made in Plexiglas, is transparent in order to allow for the optical visualization of the cavitation on the test inducer. It can easily be replaced allowing for tests on inducers with different tip diameters and clearances.

The facility is instrumented with a series of transducers for measuring:

- the inducer inlet pressure;

- the inducer static pressure rise;

- the pressure fluctuations, in order to identify the presence of flow instabilities;

- the volumetric flow rate on the suction line;

- the volumetric flow rate on the discharge line;

- the fluid temperature inside the main tank;

- the fluid temperature at the inducer inlet;

- the absolute angular position of the driving shaft;

- the absolute angular position of the eccentric shaft which generates the whirl motion.
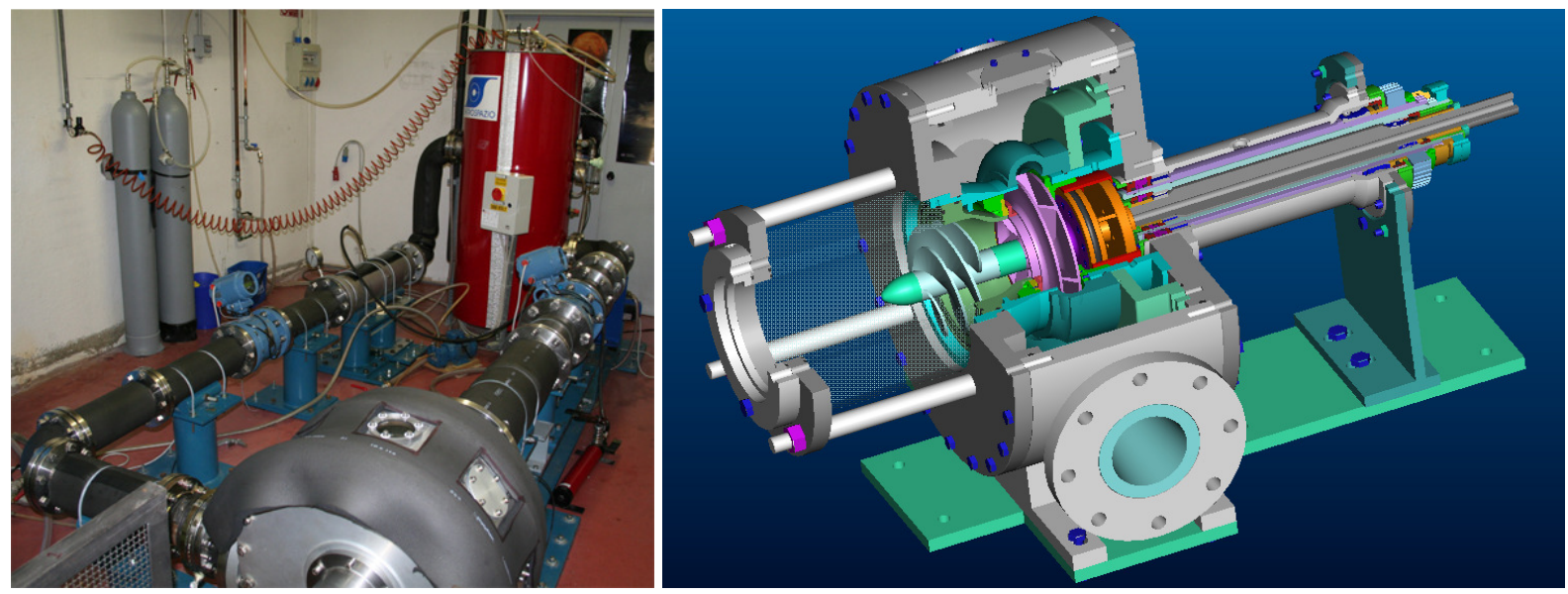

Figure 1. The Cavitating Pump Rotordynamic Test Facility (left) and cut-off drawing of the CPRTF test section (right), where the orange component is the rotating dynamometer. 
The test section is equipped with a rotating dynamometer, for the measurement of the forces and moments acting on the impeller (Figure 1, right).

The generation of the eccentricity is based on a two-shafts mechanism. The shafts are mounted one inside the other and the eccentricity can be regulated in the range between 0 and $2 \mathrm{~mm}$ by means of two eccentric holes, whose relative angular position can be finely adjusted from 0 to $2 \mathrm{~mm}$ before each rotordynamic test. The whirl motion is generated by a brushless motor driving the external shaft, while the impeller rotation is imparted by connecting the internal shaft to the main motor with an omokinetic coupling.

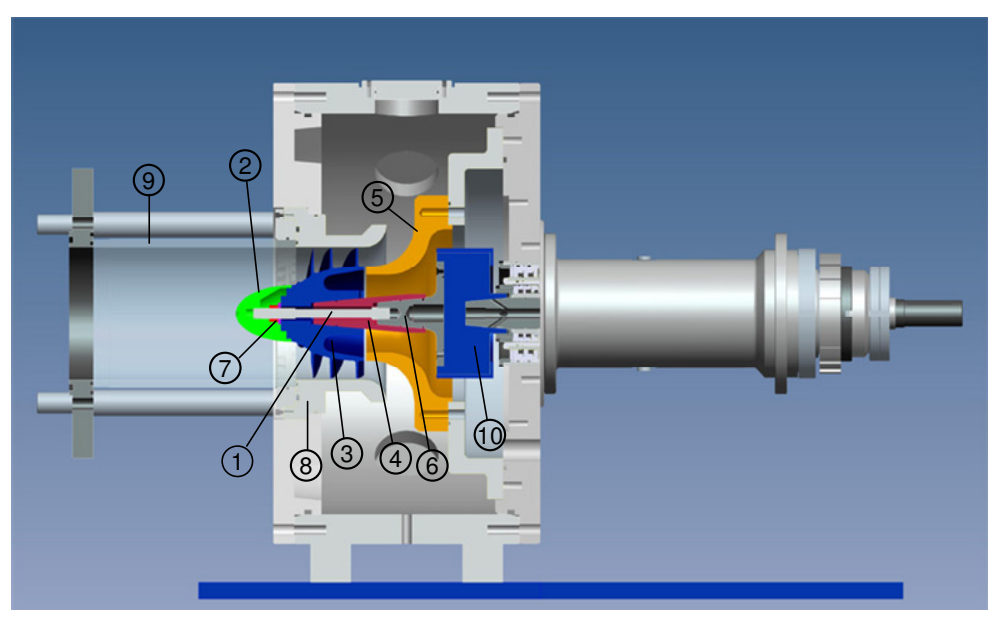

Figure 2. Rendering of the test chamber assembly in the rotordynamic configuration (CPRTF) used for the test campaign reported in the present paper.
The rotating dynamometer is realized in one single piece of phase hardening steel AISI $630 \mathrm{H} 1025$ and consists of two flanges connected by four square cross-section posts acting as flexible elements. The deformation of the posts is measured by 40 semiconductor strain gauges arranged in 10 full Wheatstone bridges, which provide redundant measurements of the forces and moments acting on the impeller. Each bridge is temperature self-compensated, with separate bipolar excitation and read-out for better reduction of cross-talking. The sizing of the sensing posts is the result of a tradeoff between sensitivity and structural resistance, operational stability and position control (stiffness). The current design of the dynamometer is optimized for a suspended mass of $4 \mathrm{~kg}$ with 70

$\mathrm{mm}$ gyration radius, an added mass of about $2 \mathrm{~kg}$ (based on the expected magnitude of the rotordynamic forces), a rotational speed of $3000 \mathrm{rpm}$ without eccentricity, and maximum rotational and whirl speeds up to $2000 \mathrm{rpm}$ with 2 mm shaft eccentricity.

Figure 2 shows a rendering of the test chamber assembly used for the experimental campaign reported in the present paper. The rotating dynamometer (part 10) is placed between the inducer and the driving shaft. The inducer is therefore suspended for the measurement of the rotor forces. In order to reduce the cantilever effects, the inducer has been recessed with respect to the optical access to the test section inlet. In this configuration the inducer blades are contained within the "inlet duct" (part 8). A nominal clearance of $2 \mathrm{~mm}$ allows for accommodating whirl eccentricities sufficiently large for generating measurable rotordynamic forces. The inducer is connected to the dynamometer (part 6) by means of a male/female conical endings adapter (part 4). A screwed component (part 1) is coupled on one side with a threaded hole machined in the conical interface of the dynamometer and on the other side with a nut (part 7).

The relative positions of the inducer (part 3) on the adapter (part 4) and of the adapter on the dynamometer interface (part 6) are guaranteed by means of two pins. The "radial diffuser" (part 5) has been mounted on the test section in order to reduce the suspended mass and minimize the interference with the measurement of the forces acting on the inducer.

In order to avoid water sloshing effects in the inducer assembly (e.g. within the nose (part 2), between the inducer and part 1 or between part 1 and part 4) the upstream nose and the conical mounts of the inducer have been sealed by means of o-rings.

\section{B. Test Article}

The experimental campaign has been conducted on a four-bladed, tapered-hub, variable-pitch inducer, named DAPAMITO4. The test inducer, whose main geometrical and operational parameters are reported in Table 1, is made of 7075-T6 aluminum alloy and has been designed by means of the reduced order model described in d'Agostino et al. ${ }^{11,12}$.

The overall dimensions of this inducer have been chosen for installation and testing in the current CPRTF configuration. A moderate value of the blade loading (with a diffusion factor $D=0.38$ as defined in d'Agostino et al. $\left.{ }^{11,12}\right)$ and a high solidity $\left(\sigma_{T}=2.25\right)$ have been chosen for reducing the leading-edge cavity and improving the 
suction performance. The value of the tip incidence-to-blade angle ratio $\alpha / \beta_{b}<0.5$ has been selected with the aim of controlling the danger of surge instabilities at design flow under cavitating conditions.

Table 1. Geometrical and operational parameters of the DAPAMITO4 inducer.

\begin{tabular}{|l|c|c|c|}
\hline Design flow coefficient & {$[--]$} & $\Phi_{D}$ & 0.070 \\
\hline Number of blades & {$[--]$} & $N$ & 4 \\
\hline Tip radius & $\mathrm{mm}$ & $r_{T}$ & 81.0 \\
\hline Inlet tip blade angle & $\mathrm{deg}$ & $\gamma_{T l e}$ & 81.10 \\
\hline Inlet hub radius (fully-developed blade) & $\mathrm{mm}$ & $r_{H l e}$ & 48.0 \\
\hline Outlet hub radius & $\mathrm{mm}$ & $r_{H t e}$ & 58.5 \\
\hline Mean blade height & $\mathrm{mm}$ & $h_{m}$ & 27.75 \\
\hline Axial length (fully-developed blade) & $\mathrm{mm}$ & $c_{a}$ & 63.5 \\
\hline Rotational speed & $\mathrm{rpm}$ & $\Omega$ & 3000 \\
\hline Inlet hub radius & $\mathrm{mm}$ & $r_{H 1}$ & 35.0 \\
\hline Axial length & $\mathrm{mm}$ & $L$ & 90.0 \\
\hline \hline Diffusion factor & {$[--]$} & $D$ & 0.38 \\
\hline Ratio between tip incidence and blade angles & {$[--]$} & $\alpha / \beta_{b}$ & 0.3 \\
\hline Tip solidity & {$[--]$} & $\sigma_{T}$ & 2.25 \\
\hline Incidence tip angle @ design & $\mathrm{deg}$ & $\alpha$ & 2.31 \\
\hline Outlet tip blade angle & $\mathrm{deg}$ & $\gamma_{T t e}$ & 72.46 \\
\hline
\end{tabular}

\section{Experimental Procedure}

The main objective of the experiments was the measurement of the steady (lateral) and unsteady (rotordynamic) forces on the DAPAMITO4 inducer. The six components of the generalized force vector acting on the inducer have been measured by means of the rotating dynamometer. Data acquisition has been designed for easy correlation of the inducer position with the measured forces.

In the experiments the rotating velocities of the main and whirl motors, $\Omega$ and $\omega$ respectively, are electrically controlled to have a common minimum integer multiple $(\Omega / J=\omega / I)$ for the orientation of the dynamometer and its location on the whirl orbit to return back to their original positions after each period of the reference frequency $\Omega / J$ $=\omega / I^{4,5,8,9}$.

\section{A. Data Reduction Procedure}

The generic components of the instantaneous forces acting on a whirling inducer are schematically shown in Figure 3. The instantaneous force vector $\vec{F}$ can be expressed as the sum of a steady force $\vec{F}_{0}$ (not depending on the presence of a whirl motion) and an unsteady force related to the perturbation vector $\vec{\varepsilon}$ by means of the rotordynamic matrix $\boldsymbol{A}$ :

$$
\left(\begin{array}{c}
F_{X} \\
F_{Y}
\end{array}\right)=\left(\begin{array}{c}
F_{0 X} \\
F_{0 Y}
\end{array}\right)+\left[\begin{array}{ll}
A_{X X} & A_{X Y} \\
A_{Y X} & A_{Y Y}
\end{array}\right]\left(\begin{array}{l}
\varepsilon \cos \left(\omega t+\omega_{0}\right) \\
\varepsilon \sin \left(\omega t+\omega_{0}\right)
\end{array}\right)
$$

where $\omega_{0}$ is the anomaly of the eccentricity at the initial acquisition time $t_{0}$.

Since the rotordynamic matrix $\boldsymbol{A}$ expresses a (rotational) transformation between two coplanar vectors, its elements satisfy the symmetry conditions $A_{X X}=A_{Y Y}$ and $A_{X Y}=-A_{Y X}$. The relationship between the forces in the rotating reference frame, $F_{x}(t)$ and $F_{y}(t)$, and the forces in the laboratory (stationary) reference frame can be expressed as:

$$
\begin{aligned}
& F_{X}(t)=F_{x}(t) \cos \left(\Omega t+\Omega_{0}\right)-F_{y}(t) \sin \left(\Omega t+\Omega_{0}\right) \\
& F_{Y}(t)=F_{x}(t) \sin \left(\Omega t+\Omega_{0}\right)+F_{y}(t) \cos \left(\Omega t+\Omega_{0}\right)
\end{aligned}
$$


where $\Omega_{0}$ represents the angular position of the rotating axis $(x)$ w.r.t. the absolute one $(X)$ at the initial acquisition time $t_{0}$. The relationship between the forces in the rotating frame, $F_{x}(t)$ and $F_{y}(t)$, the steady force components $F_{0 X}$ and $F_{0 Y}$, and the rotordynamic matrix $\boldsymbol{A}$ can be obtained by equating equation (1) and equation (2):

$$
\begin{aligned}
& F_{x}(t) \cos \left(\Omega t+\Omega_{0}\right)-F_{y}(t) \sin \left(\Omega t+\Omega_{0}\right)=F_{0 X}+A_{X X} \varepsilon \cos \left(\omega t+\omega_{0}\right)+A_{X Y} \varepsilon \sin \left(\omega t+\omega_{0}\right) \\
& F_{x}(t) \sin \left(\Omega t+\Omega_{0}\right)+F_{y}(t) \cos \left(\Omega t+\Omega_{0}\right)=F_{0 Y}+A_{Y X} \varepsilon \cos \left(\omega t+\omega_{0}\right)+A_{Y Y} \varepsilon \sin \left(\omega t+\omega_{0}\right)
\end{aligned}
$$

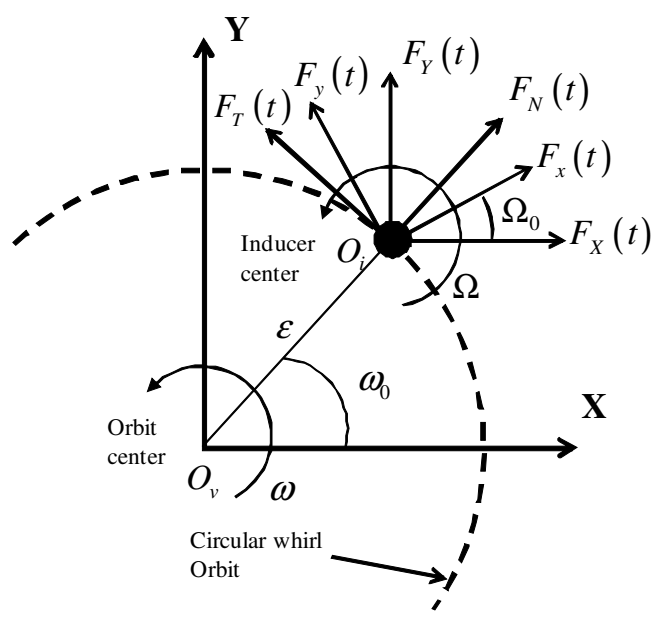

Figure 3. Schematic representation of the rotordynamic forces in the laboratory and rotating reference frames.
The steady force components, $F_{0 X}$ and $F_{0 Y}$, can be obtained as time averages, respectively, of equation (3) and (4). The matrix elements $A_{X X}$ and $A_{Y X}$ are obtained integrating equations ( 3 ) and (4) as follows:

$$
\frac{2}{T} \int_{0}^{T}[] \cos \left(\omega t+\omega_{0}\right) d t
$$

whereas the elements $A_{X Y}$ and $A_{Y Y}$ are obtained integrating equations (3) and (4) as follows:

$$
\frac{2}{T} \int_{0}^{T}[] \sin \left(\omega t+\omega_{0}\right) d t
$$

The normal and tangential forces with respect to the whirl orbit, $F_{N}$ and $F_{T}$, can be expressed as:

$$
\begin{aligned}
& F_{N}=\frac{1}{2}\left(A_{X X}+A_{Y Y}\right)=A_{X X}=A_{Y Y} \\
& F_{T}=\frac{1}{2}\left(A_{Y X}-A_{X Y}\right)=-A_{X Y}=A_{Y X}
\end{aligned}
$$

The normal force $F_{N}$ is assumed positive when in the outward direction, while $F_{T}$ is positive if it has the same direction of the rotational speed $\Omega$. Therefore a positive tangential force is destabilizing when the directions of the whirl motion and the shaft rotational motion are the same (i.e., the whirl/shaft speed ratio is positive) and stabilizing for a negative value of the whirl/shaft speed ratio. The normal force, conversely, is destabilizing when it is positive and tends to increase the radius of the whirl orbit.

The data presented in this paper are referred to purely fluid-induced forces; the effect of buoyancy and tare forces (the submerged weight and the centrifugal whirl force) have been subtracted from the total force. The steady and the unsteady forces have been obtained as mean values over 250 complete periods of the fundamental reference frequency.

The unsteady rotordynamic forces have been normalized as follows ${ }^{8,9}$ :

$$
F_{N}^{*}=\frac{F_{N}}{\pi \rho c_{a} \varepsilon \Omega^{2} r_{T}^{2}} \quad F_{T}^{*}=\frac{F_{T}}{\pi \rho c_{a} \varepsilon \Omega^{2} r_{T}^{2}}
$$

where $\rho$ is the fluid temperature, $c_{a}$ is the axial length of the inducer blades, $\varepsilon$ is the radius of the circular whirl orbit (eccentricity), $\Omega$ is the inducer rotational speed and $r_{T}$ is the inducer tip radius.

\section{B. Test Matrix}

A comprehensive experimental campaign has been carried out on the DAPAMITO4 inducer aimed at understanding the role of :

- the flow coefficient (noncavitating/cavitating regime),

- the fluid temperature (noncavitating/cavitating regime),

- the occurrence of cavitation, 


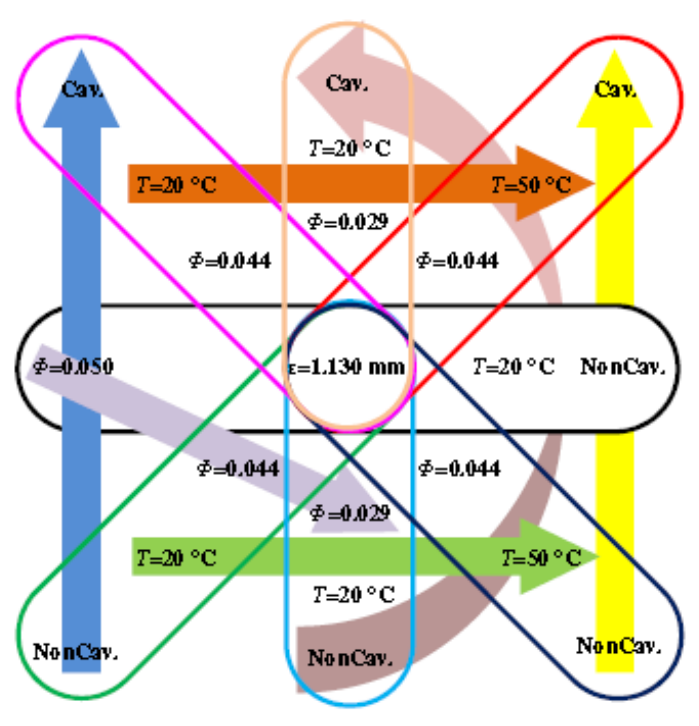

Figure 4. Test matrix.

on the rotordinamic forces. All the runs have been performed at $\Omega=1750 \mathrm{rpm}$, which represents a compromise value between the generation of measurable rotordynamic forces and the structural integrity of the rotating dynamometer. Only subsynchronous whirl ratios have been investigated, with both positive and negative values $(\omega / \Omega= \pm 0.1, \pm 0.2$, $\pm 0.3, \pm 0.5, \pm 0.7$ ).

The eccentricity has been fixed at $\varepsilon=1.130 \mathrm{~mm}$ for all the tests.

The effect of the flow rate on the rotordynamic forces has been studied under noncavitating conditions at three flow coefficients, $\Phi=0.050, \Phi=0.044$ and $\Phi$ $=0.029$ and under cavitating operation at $\Phi=0.044$ and $\Phi=0.029$.

The tests at $\Phi=0.044$ have been performed at two different water temperatures: about $20^{\circ} \mathrm{C}$ and about 50 ${ }^{\circ} \mathrm{C}$.

Figure 4 summarizes the test matrix: the arrows indicate the parameters which are changing considering two different sets of experiments contained inside the ellipsoidal areas.

\section{Results and Discussion}

\section{A. Noncavitating Performance and Hydraulic Efficiency}

A series of tests has been performed on the DAPAMITO4 inducer aimed at characterizing the noncavitating performance and the hydraulic efficiency at $18.8{ }^{\circ} \mathrm{C}$ and $49.6{ }^{\circ} \mathrm{C}$ in the same assembly configuration used for the rotordynamic tests $(2 \mathrm{~mm}$ blade tip clearance, corresponding to $6.8 \%$ of the mean blade height, and zero eccentricity).

Figure 5 shows the noncavitating experimental curves in terms of the static head coefficient $\Psi=\Delta p / \rho \Omega^{2} r_{T}^{2}$ and the hydraulic efficiency $\eta=Q \Delta p_{t} /(\tau \Omega)$ as functions of the flow coefficient $\Phi=Q / \pi \Omega r_{T}^{3}$.

The volumetric flow rate $(Q)$ has been measured by means of an electromagnetic flowmeter (mod. $8732 \mathrm{C}$ by Fisher-Rosemount, range $0-1001 / \mathrm{s}, 0.5 \%$ precision) mounted on the discharge line, the static pressure increase $(\Delta p)$ by means of a differential pressure transducer (Kulite, model BMD 1P 1500 100, range 0-6.9 bard, 0.1\% precision). The low pressure tap has been located on the suction line about 6 inducer diameters upstream of the blade leading edges, in order to eliminate the effect of inlet flow prerotation. The high pressure tap has been mounted on the discharge line at about 2.5 duct diameters downstream of the test chamber connection, because of the uncertainties in compensating for the influence of exit flow swirl if mounted on the inducer discharge casing. Hence head measurements include the losses due to the flow diffusion from the inducer outlet into the test section and to the entrance in the discharge line. In order to evaluate the total pressure rise, some assumptions have been made both on the measured static pressure rise and the dynamic pressure at the exit of the inducer.

It has been assumed that a good estimation of the bulk static pressure at the exit of the inducer can be the pressure measured in the discharge line because the losses due to the entrance in the discharge line can be considered negligible and the dynamic pressure at the exit of the inducer completely lost in the sudden diffusion into the test section. The total pressure rise across the inducer consists of the static pressure rise and the dynamic pressure rise:

$$
\Delta p_{t}=\Delta p+\frac{1}{2} \rho\left(\bar{v}_{2 \delta^{\circ}}^{2}+w_{2}^{2}-w_{1}^{2}\right)
$$

where the inlet velocity has been assumed to have only the axial component:

$$
w_{1}=\frac{Q}{A_{1}}=\frac{Q}{\pi r_{T}^{2}} \cong \text { constant }
$$

while the outlet velocity consists of two components: one axial, $w_{2}$, and the other azimuthal, $v_{2 \delta^{\circ}}$. As a first approximation, the outlet axial velocity can be computed as follows: 


$$
w_{2}=\frac{Q}{A_{2}}=\frac{Q}{\pi\left(r_{T}^{2}-r_{H 2}^{2}\right)} \cong \text { constant }
$$

while the contribution of the azimuthal velocity, $v_{2 \delta^{\circ}}$, to the dynamic pressure should be integrated in the outlet section:

$$
\bar{v}_{2 \delta^{\circ}}^{2}=\frac{1}{A_{2}} \int_{r_{H 2}}^{r_{T}} 2 \pi r v_{2 \delta^{\circ}}^{2} d r
$$

A good approximation of the azimuthal velocity can be obtained by using the deviation angle, $\delta=\beta_{2}-\gamma_{2}$, computed by means of the Carter's rule:

$$
v_{2 \delta^{\circ}}=\Omega r-w_{2} \tan \left(\gamma_{2}+\delta^{\circ}\right)=\left[\Omega-\frac{2 \pi w_{2}}{\tilde{P}}\right] r
$$

where:

$$
\tilde{P}=\frac{2 \pi r}{\tan \left(\gamma_{2}+\delta^{\circ}\right)}=\frac{2 \pi r_{T}}{\tan \left(\gamma_{T t e}+\delta_{T t e}^{\circ}\right)} \quad \text { and } \quad \delta_{T t e}^{\circ} \cong \frac{\left[0.23+0.1\left(\frac{\gamma_{T t e}}{50^{\circ}}\right)\right]}{\sqrt{\sigma}}\left(\gamma_{T l e}-\gamma_{T t e}\right)
$$

Finally, the contribution to the dynamic pressure related to the azimuthal velocity can be estimated by means of the following expression:

$$
\bar{v}_{2 \delta^{\circ}}^{2}=\frac{1}{A_{2}} \int_{r_{H 2}}^{r_{T}} 2 \pi r v_{2 \delta^{\circ}}^{2} d r=\frac{\left(r_{T}^{4}+r_{H 2}^{4}\right)}{2}\left[\Omega-\frac{2 \pi w_{2}}{\tilde{P}}\right]^{2}
$$

The torque $(\tau)$ has been directly measured by means of the rotating dynamometer, thereby by-passing the uncertainties associated with seal friction on the inducer shaft.

The use of the same material (aluminum) for both the inducer and its casing minimizes the relative changes of the tip clearance due to differential thermal expansion in tests at elevated liquid temperature. The curves, obtained at a rotational speed of $1750 \mathrm{rpm}$ and several temperatures of the liquid, confirm the independence of the test results on the flow temperature.

The highest measured efficiency corresponds to the highest experimental flow coefficient $(\Phi=0.052)$ is about $77 \%$, in accordance with the typical values for this kind of machine $(70 \div 80 \%)$.

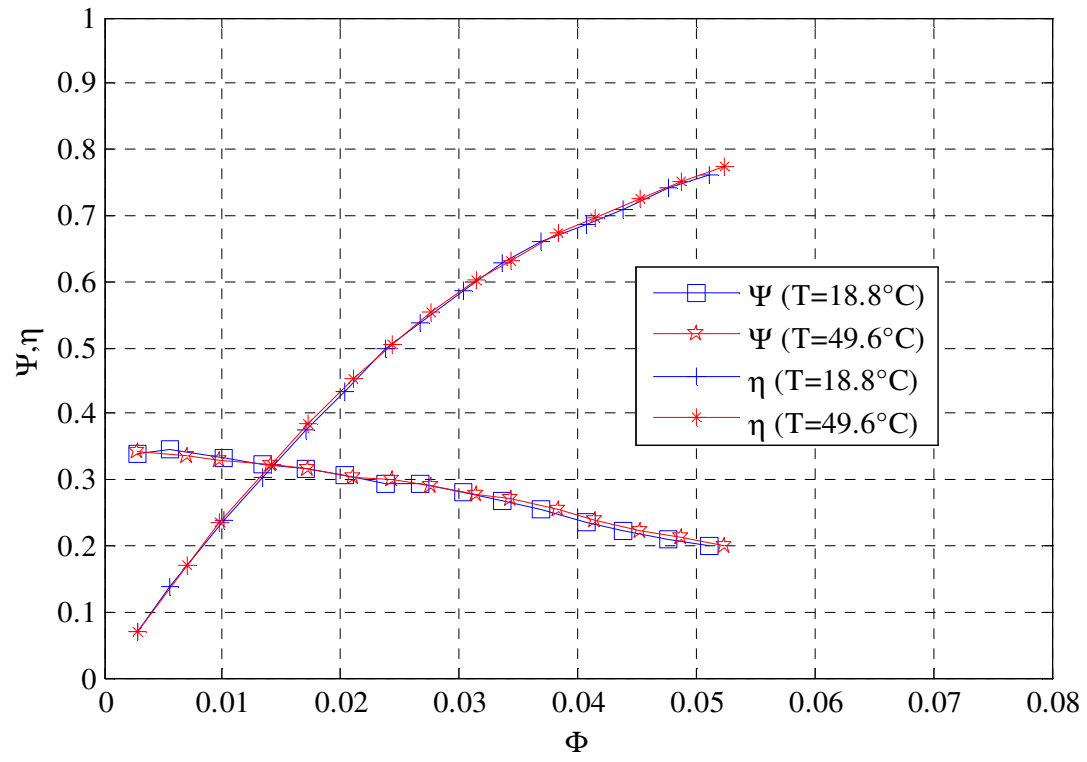

Figure 5. DAPAMITO4 inducer noncavitating performance and hydraulic efficiency at different water temperatures. 


\section{B. Rotordynamic Forces}

Classically $^{4,5,8,9}$, rotordynamics is the study of the unsteady normal and tangential forces, and their destabilizing/stabilizing effects on the whirl motion are determined by whether the orientation of the normal $\left(\vec{F}_{N}^{*}\right)$ and tangential $\left(\vec{F}_{T}^{*}\right)$ components of the rotordynamic force $\left(\vec{F}_{R}^{*}\right)$ tend to promote the increase of the whirl eccentricity or to sustain its rotation. As already pointed out in Section III.A (see Figure 6), the normal component is destabilizing when directed outward, while the tangential component is destabilizing when directed in the same direction as the whirl motion. In the present paper, rotordynamic forces are reported both in term of $F_{N}^{*}$ and $F_{T}^{*}$ and in terms of their non-dimensional modulus $\left|\vec{F}_{R}^{*}\right|=\sqrt{\left(F_{N}^{*}\right)^{2}+\left(F_{T}^{*}\right)^{2}}$ and their phase angle, $\phi$, w.r.t. the eccentricity vector ( $\vec{\varepsilon}$ ), as functions of the whirl ratio $\omega / \Omega$.
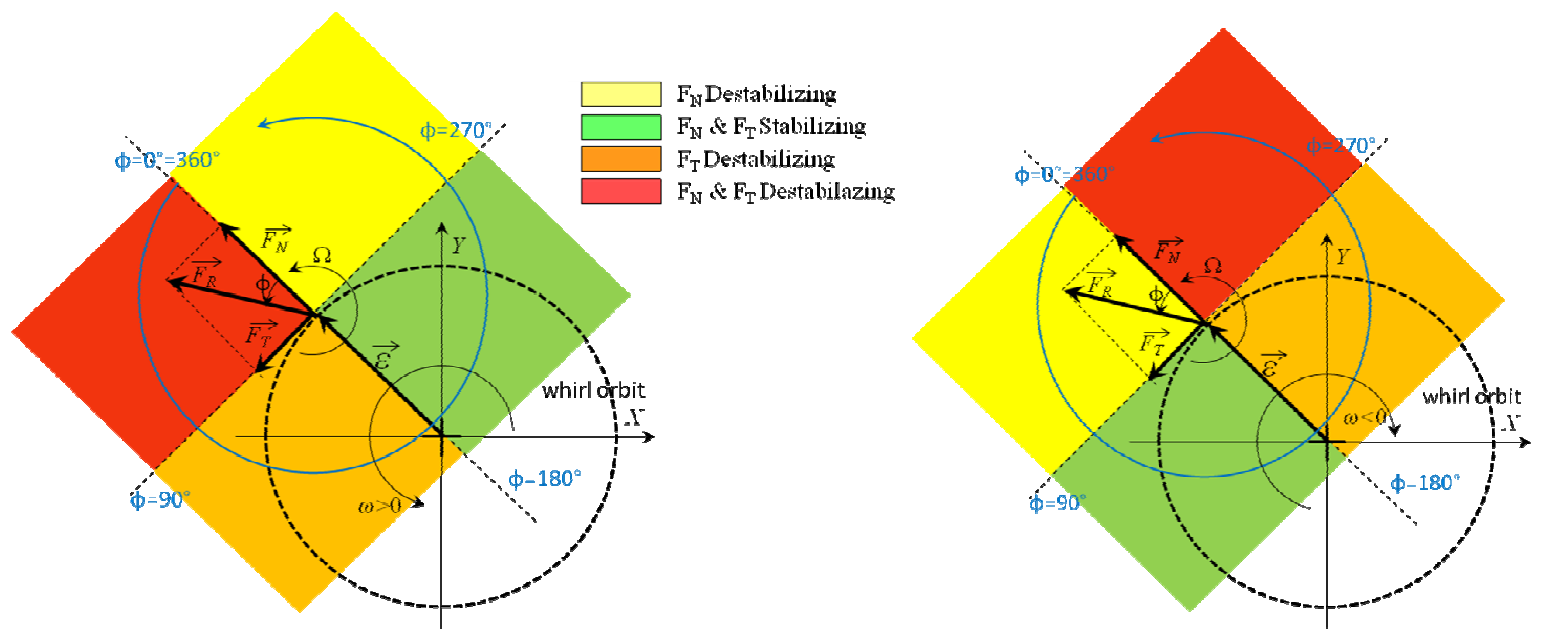

Figure 6. Schematic of the decomposition of the rotordynamic force $\left(\vec{F}_{R}\right)$ in its components normal $\left(\vec{F}_{N}\right)$ and tangent $\left(\vec{F}_{T}\right)$ to the whirl orbit. The convention adopted for the phase angle $(\phi)$ between the rotordynamic force $\left(\vec{F}_{R}\right)$ and the eccentricity $(\vec{\varepsilon})$ is shown: coloured areas refer to the different stability regions for positive (left) and negative (right) whirl ratio.

The same figure shows, as green, yellow, orange and red areas, the stability regions for positive (left) and negative (right) whirl ratios: the same colors have been used in the phase charts for highlighting the behavior of the rotordynamic forces. This way of representing the rotordynamic forces gives direct information about the amplitude and direction (and therefore the stabilizing/destabilizing behavior) of the mean value of the unsteady force acting on the inducer.

The analysis of the rotordynamic forces in noncavitating conditions has been carried out for relative low values of the flow coefficient $\left(\Phi<71 \% \Phi_{D}\right)$, all characterized by the occurrence of flow reversal.

Figure 7 and Figure 8 show the discrete spectra of the rotordynamic forces as functions of the whirl ratio for three values of the flow coefficient in water at ambient temperature under noncavitating conditions.

As for a three-bladed inducer tested at ALTA in a previous experimental campaign (Torre et al. ${ }^{13}$ ), in general the magnitude of the rotordynamic force tends to increase as the flow coefficient decreases (see Figure 8). Furthermore, the spectrum qualitatively retains the same shape, except for a translation towards higher values of the whirl ratio for lower flow coefficients.

For negative whirl ratios (see Figure 7 and Figure 8) there is a threshold value beyond which the normal component of the rotordynamic force becomes destabilizing. This threshold value, $\omega / \Omega \leq-0.3$, is almost the same for all the flow coefficients that have been investigated.

For positive whirl ratios the normal rotordynamic force is always stabilizing, while the tangential force component exhibits destabilizing values in correspondence of whirl ratio ranges that strongly depend on the flow coefficient. In general, three different intervals have been observed: for low values of the whirl ratio the tangential force is 
destabilizing; for intermediate values it becomes stabilizing; at higher whirl ratios it is again destabilizing. The boundaries of these intervals are influenced by the flow coefficient and the general trend is a shift towards higher values of the whirl ratio when the flow coefficient decreases, as also observed in a previous test campaign on a three-bladed inducer ${ }^{13}$.
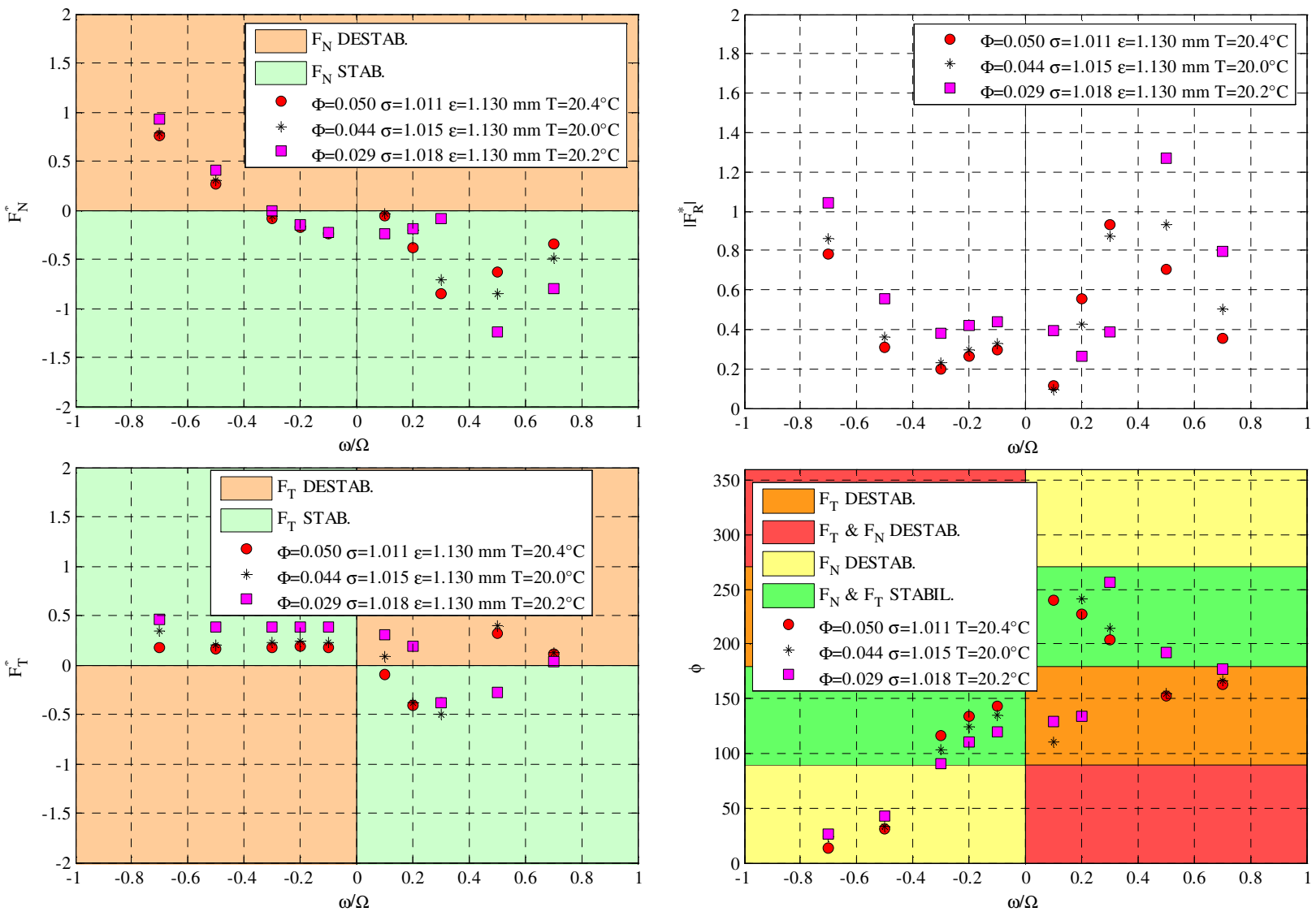

Figure 7. Effect of the flow coefficient $\Phi$ on the normal $\left(F_{N}^{*}\right)$ and tangential $\left(F_{T}^{*}\right)$ components of the rotordynamic force: noncavitating regime.

Figure 8. Effect of the flow coefficient $\Phi$ on the intensity $\left(\left|F_{R}^{*}\right|\right)$ and phase $(\phi)$ of the rotordynamic force: noncavitating regime.

In order to investigate the effect of the fluid temperature on the rotordynamic forces under noncavitating conditions, the water temperature has been increased up to $50.3{ }^{\circ} \mathrm{C}$. Figure 9 shows the intensity and phase charts for a series of tests carried out at different temperatures, maintaining a constant flow coefficient $\Phi=0.044$. The plots show good agreement between the results at different temperatures, which is even more evident when the results are reported in terms of the normal and tangential components $F_{N}^{*}$ and $F_{T}^{*}$ of the rotordynamic force.

Cavitation plays a very important role on the rotordynamic forces and has a dramatically clear destabilizing effect, as also reported in the available literature. The comparison between cavitating and noncavitating performance in cold and hot water is reported in the figures from 11 to 16 . At $\Phi=0.044$ and ambient temperature the highest cavitation number $(\sigma=1.015)$ corresponds to a noncavitating regime, whereas the lowest one $(\sigma=0.091)$ refers to a cavitating regime with a $19 \%$ head degradation w.r.t. the noncavitating case $\left(\Psi / \Psi_{N C}=0.81\right)$.

Under cavitating conditions, the destabilizing peak at $\omega / \Omega=0.3$ is more evident in the $F_{T}^{*}$ chart (see Figure 11). 

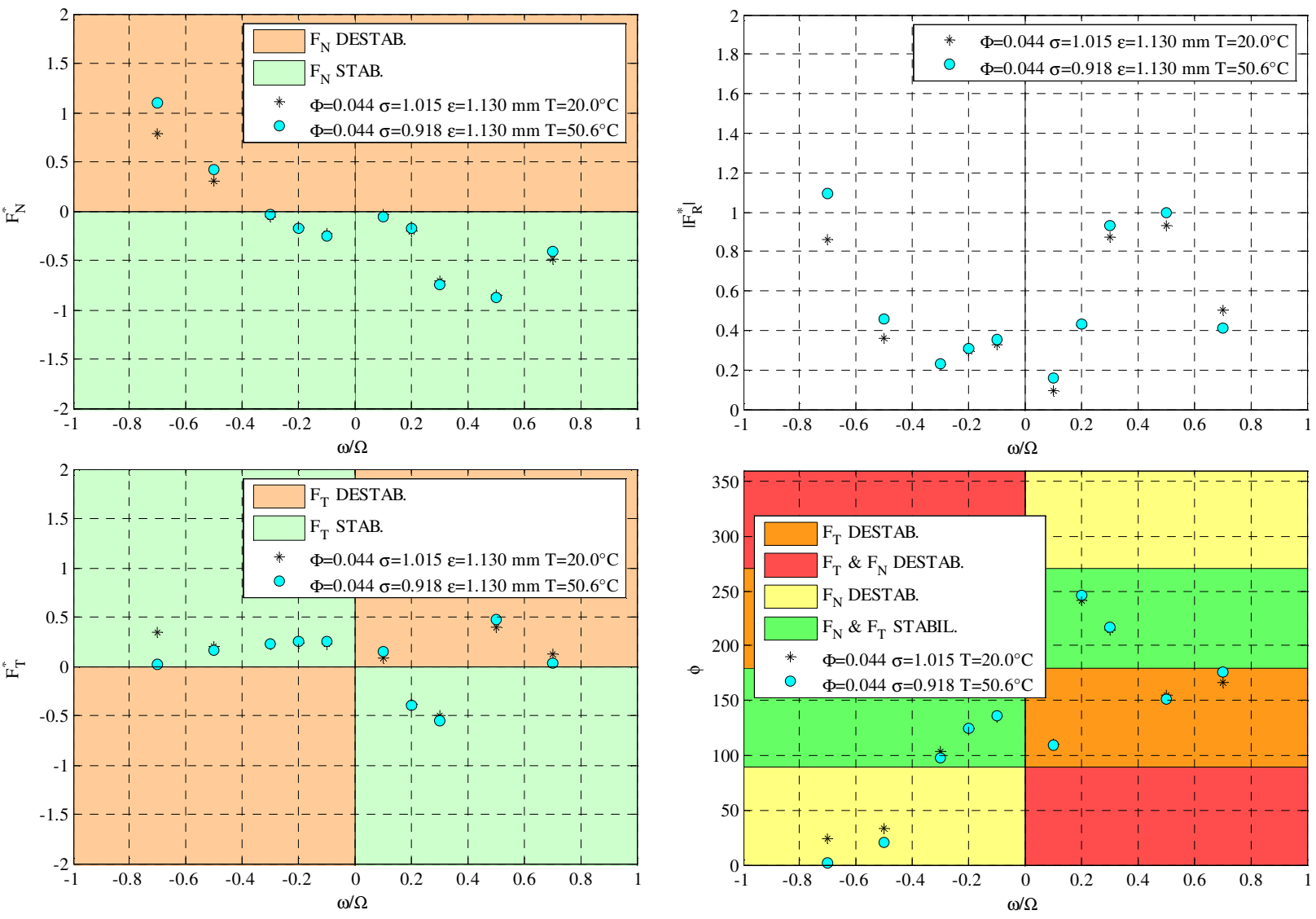

Figure 9. Effect of the fluid temperature $T$ on the normal $\left(F_{N}^{*}\right)$ and tangential $\left(F_{T}^{*}\right)$ components of the rotordynamic force: noncavitating regime.

Figure 10. Effect of the fluid temperature $T$ on the intensity $\left(\left|F_{R}^{*}\right|\right)$ and phase $(\phi)$ of the rotordynamic force: noncavitating regime.

Comparison between the cavitating and noncavitating performance at the same flow coefficient in hot water yields to the same conclusion, as reported in Figure 15 and Figure 16.

At $\Phi=0.029$ and ambient temperature, the performance degradation has been lower $\left(\Psi / \Psi_{N C}=0.91\right)$ and, consequently, the increasing trend of rotordynamic forces is less evident but, even in this case, there has been a dramatically clear destabilizing effect at $\omega / \Omega=0.5$.

Finally, Figure 17 and Figure 18 show the effect of the fluid temperature on the rotordynamic forces under developed cavitation at $\Phi=0.044$ at the same nominal cavitation number and performance degradation $\left(\Psi / \Psi_{N C}=\right.$ 0.81). The flow temperature seems to affect the rotordynamic forces in the same way as for the noncavitating regime: no significant changes on the intensity of the rotordynamic force and on its stabilizing/destabilizing behaviour are observed when the temperature is increased up to $50.1{ }^{\circ} \mathrm{C}$. This invariance of the results is probably due to the insufficiently high value of the liquid temperature for the occurrence of significant thermal cavitation effects. 

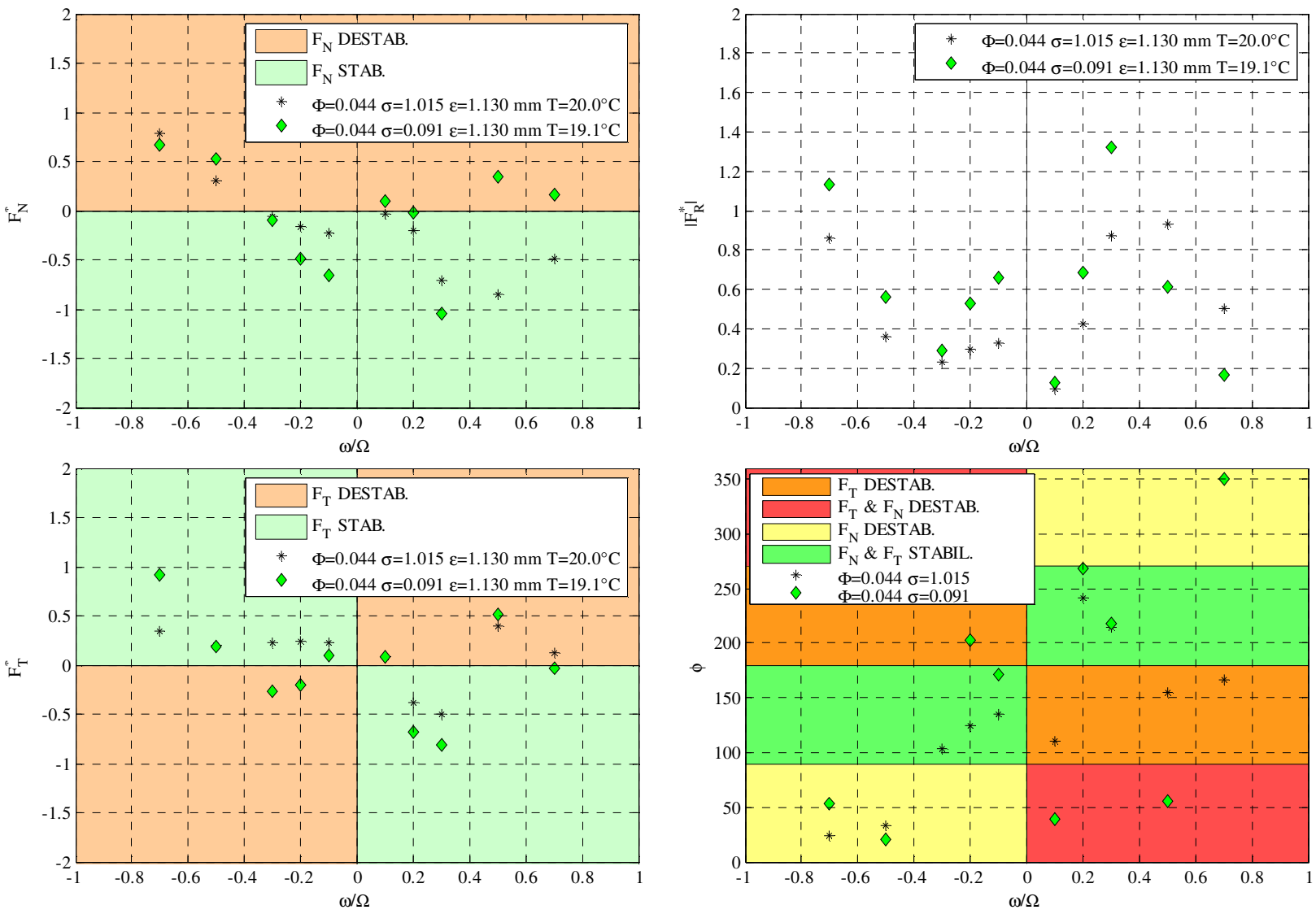

Figure 11. Effect of the cavitation number $\sigma$ on the normal $\left(F_{N}^{*}\right)$ and tangential $\left(F_{T}^{*}\right)$ components of the rotordynamic force: cold tests at $\phi=0.044$.

Figure 12. Effect of the cavitation number $\sigma$ on the intensity $\left(\left|F_{R}^{*}\right|\right)$ and phase $(\phi)$ of the rotordynamic force: cold tests at $\phi=0.044$. 

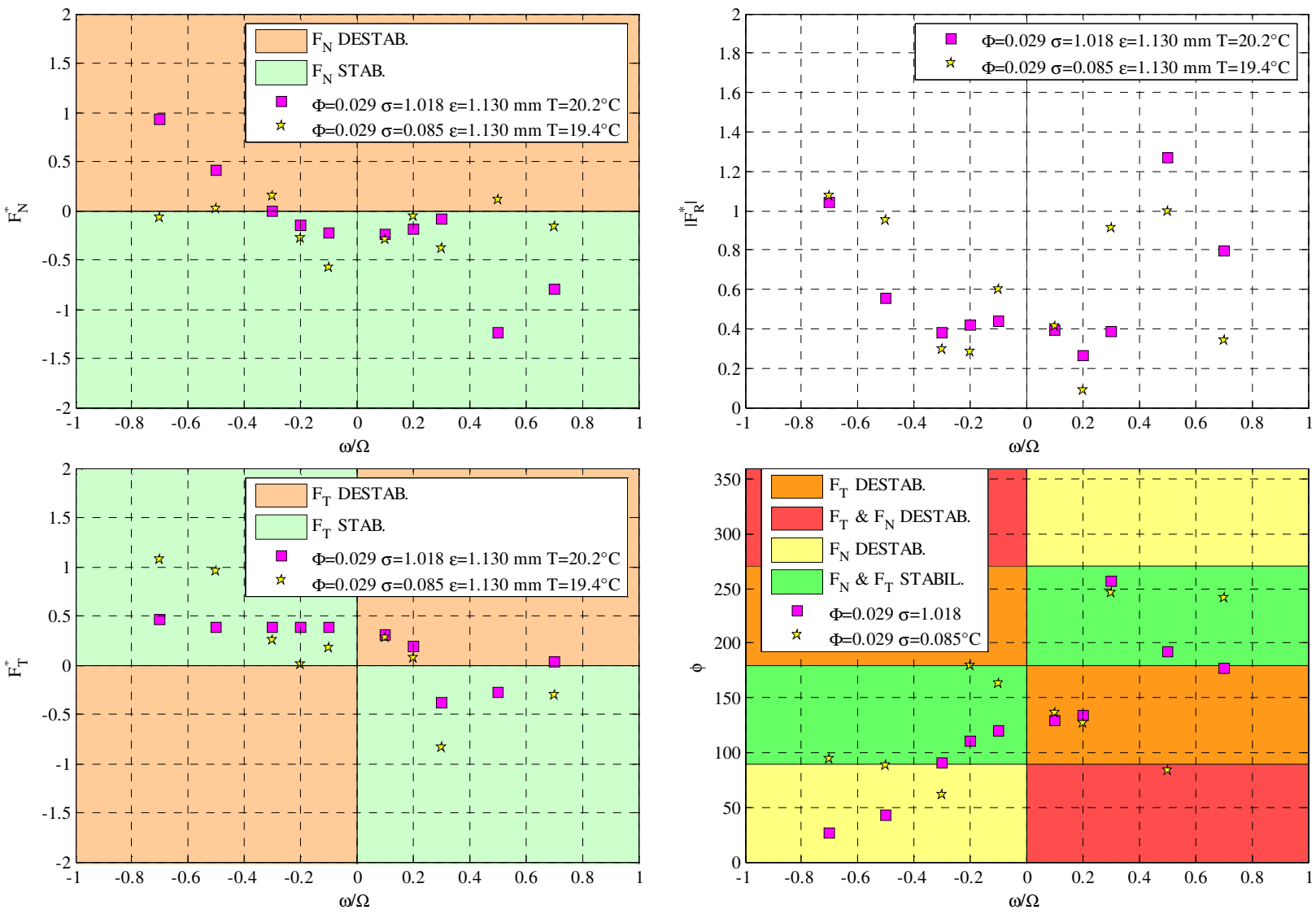

Figure 13. Effect of the cavitation number $\sigma$ on the normal $\left(F_{N}^{*}\right)$ and tangential $\left(F_{T}^{*}\right)$ components of the rotordynamic force: cold tests at $\phi=0.029$.

Figure 14. Effect of the cavitation number $\sigma$ on the intensity $\left(\left|F_{R}^{*}\right|\right)$ and phase $(\phi)$ of the rotordynamic force: cold tests at $\phi=0.029$. 

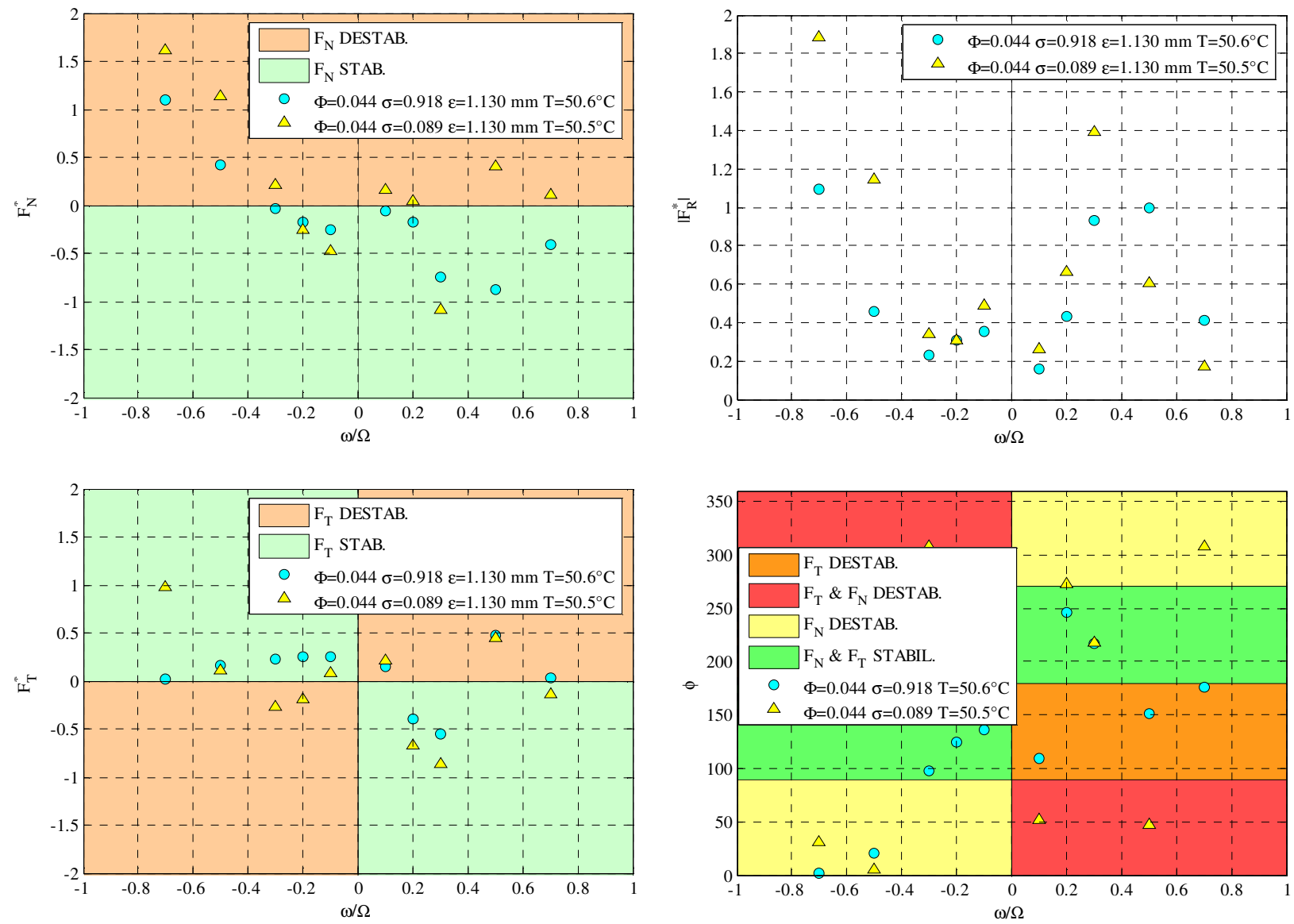

Figure 15. Effect of the cavitation number $\sigma$ on the normal $\left(F_{N}^{*}\right)$ and tangential $\left(F_{T}^{*}\right)$ components of the rotordynamic force: hot tests at $\phi=0.044$.

Figure 16. Effect of the cavitation number $\sigma$ on the intensity $\left(\left|F_{R}^{*}\right|\right)$ and phase $(\phi)$ of the rotordynamic force: hot tests at $\phi=0.044$. 

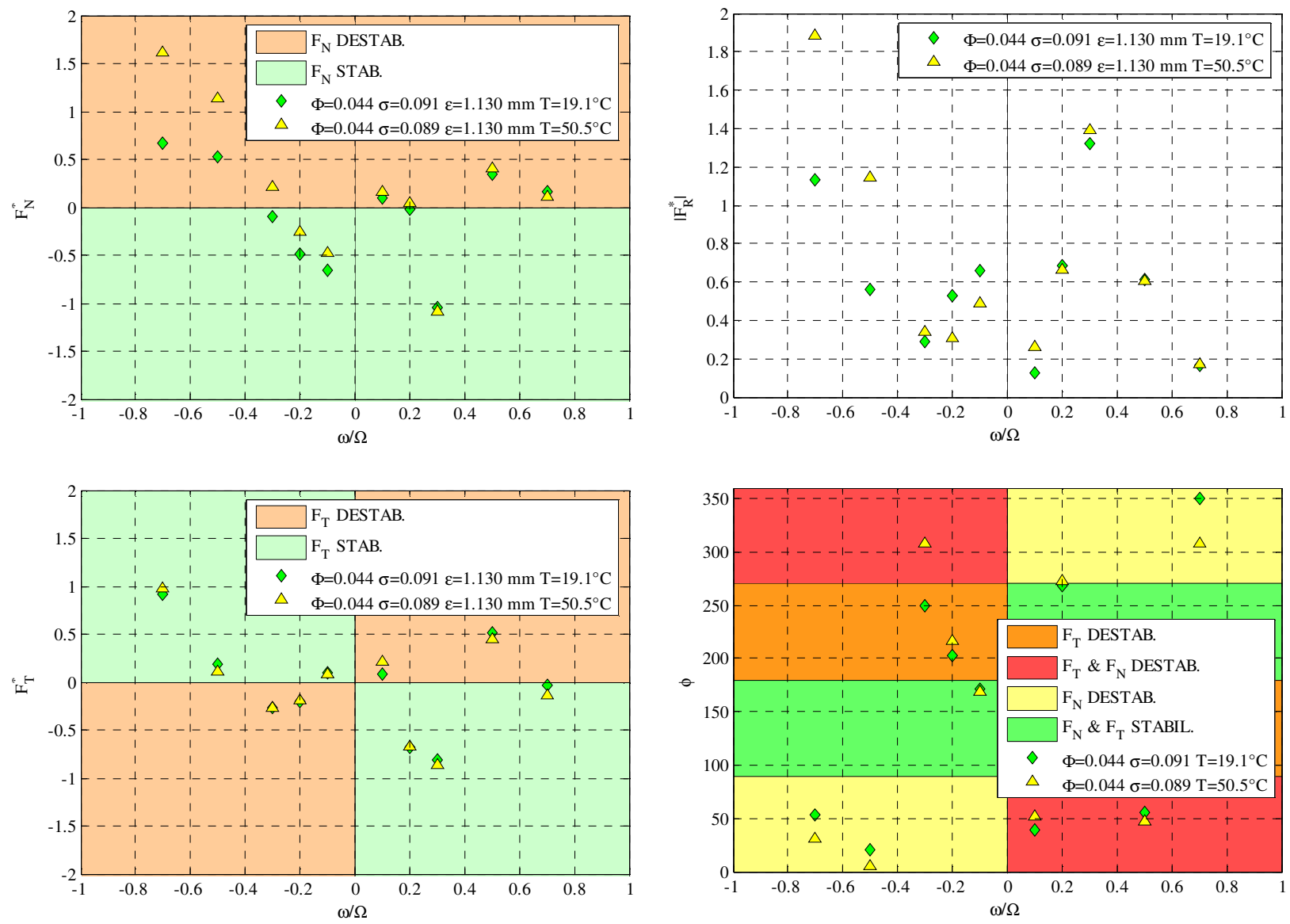

Figure 17. Effect of the fluid temperature $T$ on the normal $\left(F_{N}^{*}\right)$ and tangential $\left(F_{T}^{*}\right)$ components of the rotordynamic force: cavitating regime.

Figure 18. Effect of the fluid temperature $T$ on the intensity $\left(\left|F_{R}^{*}\right|\right)$ and phase $(\phi)$ of the rotordynamic force: cavitating regime.

\section{Conclusions}

The experimental campaign carried out on a whirling four-bladed inducer (DAPAMITO4) and reported in the present paper represents the continuation of the research work undertaken at ALTA S.p.A. with the aim of understanding the complex relationships between the fluid induced forces on inducer and its operational conditions. The role of flow coefficient, fluid temperature and cavitation on the rotordynamic forces has been investigated and the following conclusions can be drawn:

- the reduction of the flow coefficient together with the occurrence of flow reversal can dramatically change the behavior of the rotordynamic forces, which can become highly destabilizing;

- cavitation plays a very important role on the rotordynamic forces: it tends to increase their intensity and has a dramatically destabilizing effect at both positive and negative whirl ratios;

- the fluid temperature does not particularly affect the rotordynamic forces under noncavitating conditions. Furthermore, the rotordynamic forces in water do not seem to be affected by the increase of the flow temperature up to about $50{ }^{\circ} \mathrm{C}$ under cavitating conditions. This temperature value is probably too low for allowing the cavitation thermal effects to manifest themselves and affect the rotordynamic forces;

- the results obtained on the four-bladed DAPAMITO4 inducers show the same behavior of those obtained on the three-bladed DAPAMITO3 inducer, tested in a in a previous experimental campaign (Torre et al. ${ }^{13}$ ). 
The results show that the tangential and normal components of the rotordynamic forces as a function of the whirl ratio do not exhibit the quadratic functional behavior often assumed in the open literature and that destabilizing peaks can occur in particular flow conditions, potentially compromising the stability of an actual pump configuration operation.

\section{Acknowledgments}

The present work has been supported by the European Space Agency under Contract No. 20081/06/NL/IA. The authors would like to express their gratitude to Dr. Johan Steelant of ESA-ESTEC, Prof. Mariano Andrenucci and Prof. Fabrizio Paganucci of the Aerospace Department, University of Pisa, Pisa, Italy, for their constant and friendly encouragement. A special thank goes to our friend and colleague K. Shinkatsu from GMYS-Space.

\section{References}

${ }^{1}$ Ehrich, F., Childs, S. D., "Self-Excited Vibrations in High Performance Turbomachinery”, Mechanical Engineering, pp. 6679, May 1984.

${ }^{2}$ Hergt, P., Krieger, P., "Radial Forces in Centrifugal Pumps with Guide Vanes”, Proc. Inst. Mech. Eng., 184, Part 3N, pp. 101-107, 1969.

${ }^{3}$ Ohashi, H., Shoji, H., "Lateral Fluid Forces Acting on a Whirling Centrifugal Impeller in Vaneless and Vaned Diffuser", Proc. Workshop on Rotordynamic Instability Problems in High Performance Turbomachinery, NASA Conf. Publ. 2338, pp. 109122, 1984.

${ }^{4} J e r y$, B., "Experimental Study of Unsteady Hydrodynamic Force Matrices on Whirling Centrifugal Pump Impellers", Report no. 200.22, California Institute of Technology, Pasadena, USA, 1987.

${ }^{5}$ Franz, R. J., "Experimental Investigation of the Effect of Cavitation on the Rotordynamic Forces on a Whirling Centrifugal Pump Impeller", Ph.D. Thesis, California Institute of Technology, Pasadena, USA, 1989.

${ }^{6}$ Yoshida, Y., Tsujimoto, Y., Morimoto, G., Nishida, H., Morii, S., "Effects of Seal Geometry on Dynamic Impeller Fluid Forces and Moments", ASME Journal of Fluids Engineering, Vol. 125, pp. 786-795, 2003.

${ }^{7}$ Suzuki, T., Prunieres, R., Horiguchi, H., Tsukiya, T., Taenaka, Y., Tsujimoto, Y., "Measurements of Rotordynamic Forces on an Artificial Heart Pump Impeller", 23 ${ }^{\text {rd }}$ IAHR Symposium, Yokohama, Japan, October 2006.

${ }^{8}$ Bhattacharyya, A., "Internal Flows and Force Matrices in Axial Flow Inducers", Ph. D. Thesis, Report no. E249.18, California Institute of Technology, Pasadena, USA, 1994.

${ }^{9}$ Bhattacharyya, A., Acosta, A. J., Brennen, C. E., Caughey, T. K., "Rotordynamic Forces in Cavitating Inducers", ASME Journal of Fluids Engineering, Vol. 199, No. 4, pp.768-774, ISSN 0098-2202, 1997.

${ }^{10}$ Rapposelli, E., Cervone, A., d'Agostino, L., “A New Cavitating Pump Rotordynamic Test Facility”, AIAA Paper 20024285, 38th AIAA/ASME/SAE/ASEE Joint Propulsion Conference, Indianapolis, IN, USA, July 8-11, 2002.

${ }^{11}$ d'Agostino, L., Torre, L., Pasini, A., Cervone, A., 2008, "On the Preliminary Design and Noncavitating Performance of Tapered Axial Inducers", ASME Journal of Fluids Engineering, Vol. 130, Is. 11.

${ }^{12}$ d'Agostino, L., Torre, L., Pasini, A., Baccarella, D., Cervone, A., Milani, A., 2008, “A Reduced Order Model for Preliminary Design and Performance Prediction of Tapered Inducers: Comparison with Numerical Simulations", 44th AIAA/ASME/SAE/ASEE Joint Propulsion Conference, Hartford, USA.

${ }^{13}$ Torre, L., Pasini, A., Cervone, A., Pecorari, L., Milani, A., d'Agostino, L., "Rotordynamic Forces on a Three Bladed Inducer", Space Propulsion Conference, San Sebastian, Spain, May 3-6, 2010. 